\title{
Evidence of Lifshitz Transition in the Thermoelectric Power of Ultrahigh-Mobility Bilayer Graphene
}

Aditya Jayaraman,* Kimberly Hsieh, Bhaskar Ghawri, Phanibhusan S. Mahapatra, Kenji Watanabe, Takashi Taniguchi, and Arindam Ghosh

Cite This: Nano Lett. 2021, 21, 1221-1227

Read Online

\section{ACCESS | Lill Metrics \& More | 回 Article Recommendations ｜（） Supporting Information}

ABSTRACT: Resolving low-energy features in the density of states (DOS) holds the key to understanding a wide variety of rich novel phenomena in graphene-based $2 \mathrm{D}$ heterostructures. The Lifshitz transition in bilayer graphene (BLG) arising from trigonal warping has been established theoretically and experimentally. Nevertheless, the experimental realization of its effects on transport properties has been challenging because of its relatively low energy scale $(\sim 1 \mathrm{meV})$. In this work, we demonstrate that the thermoelectric power (TEP) can be used as an effective probe to investigate fine changes in the DOS of BLG. We observed additional entropy features in the vicinity of the charge neutrality point (CNP) in gapped BLG. This apparent violation of the Mott
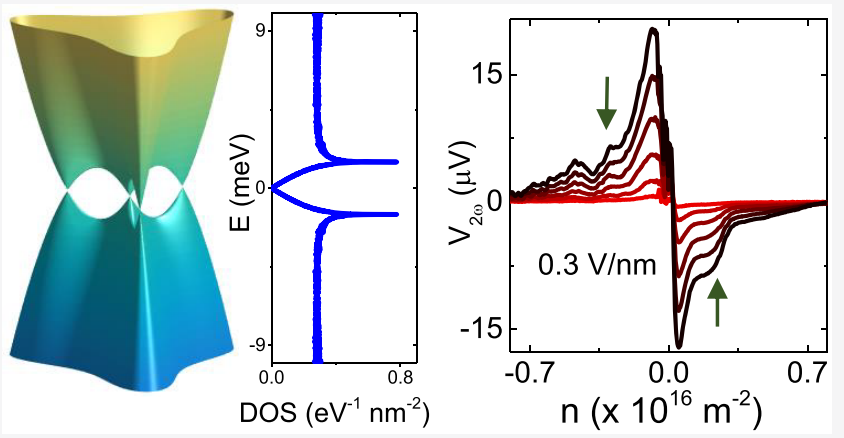
formula can be explained quantitatively by considering the effects of trigonal warping, thereby serving as possible evidence of a Lifshitz transition.

KEYWORDS: Bilayer graphene, Thermopower, Lifshitz transition, Tight binding

\section{INTRODUCTION}

The Lifshitz transition (LT) involves changes in the topology of the Fermi surface that result in anomalies in the electronic properties of a wide variety of systems. ${ }^{1}$ A LT is generally accompanied by the divergence of the density of states (DOS), resulting in van Hove singularities (vHS). Therefore, in many cases, a LT is associated with the onset of correlated electronic phases, such as superconductivity ${ }^{2,3}$ and magnetism, ${ }^{4-6}$ and plays a vital role in the properties of Weyl semimetals. ${ }^{7,8}$ In addition to the broken symmetry states, LTs also lead to modification of the transport properties at the transition. ${ }^{9,10}$ Bilayer graphene (BLG) provides a tunable test case for a LT, where the addition of the skew interlayer hopping $\left(\gamma_{3}\right)$ term (Figure 1a) in the tight-binding Hamiltonian trigonally distorts its low-energy band structure. ${ }^{11,12}$ The parabolic band structure with a single Fermi surface transforms into four Fermi surface pockets in the vicinity of $K$ and $K^{\prime}$ points (Figure $1 b)$. They coalesce to form a single Fermi surface, resulting in a LT at an energy $E_{\mathrm{L}}$ (Figure 1c).

Previous reports on the observation of $\mathrm{LT}$ in the transport properties of BLG have been scarce because of its relatively low energy scale $(\sim 1 \mathrm{meV})$, rendering these effects negligible at higher temperatures $(>10 \mathrm{~K}) \cdot{ }^{13-16}$ Furthermore, the density required to shift the chemical potential to $E_{\mathrm{L}}$ is very small $\left(n_{\mathrm{L}}\right.$ $\sim 5 \times 10^{10} \mathrm{~cm}^{-2}$ ). Hence, an experimental demonstration of such a transition is limited by fluctuation- and inhomogeneityinduced band smearing due to the presence of disorder.
Therefore, it is imperative to enhance the strength of the trigonal warping to make an experimental observation of LT feasible. Recent reports indicate that the effects of trigonal warping can be enhanced by the application of a transverse electric field $(D)$ or the presence of tensile or shear strain. ${ }^{14,17}$ In addition to the enhancement of $E_{\mathrm{L}}$, there is also a need for a measurement technique that is sensitive to the variation of the DOS.

Here, we observe the unambiguous signature of LT in BLG via the measurement of the thermoelectric power (TEP). We demonstrate the extreme sensitivity of the TEP to the band structure and that it can provide information about the electronic structure, which cannot be determined by conductance measurements alone. ${ }^{18}$ Earlier studies on the TEP of low or moderate mobility BLG have shown a general agreement with the semiclassical Mott formula ${ }^{19-22}$

$$
S_{\text {Mott }}(\mu, T)=\frac{\Delta V}{\Delta T}=-\frac{\pi^{2} k_{\mathrm{B}}^{2} T}{3 e} \frac{1}{G}\left(\frac{\mathrm{d} G}{\mathrm{~d} n} \frac{\mathrm{d} n}{\mathrm{~d} E}\right)_{E=\mu}
$$

Received: September 4, 2020

Revised: January 15, 2021

Published: January 27, 2021 

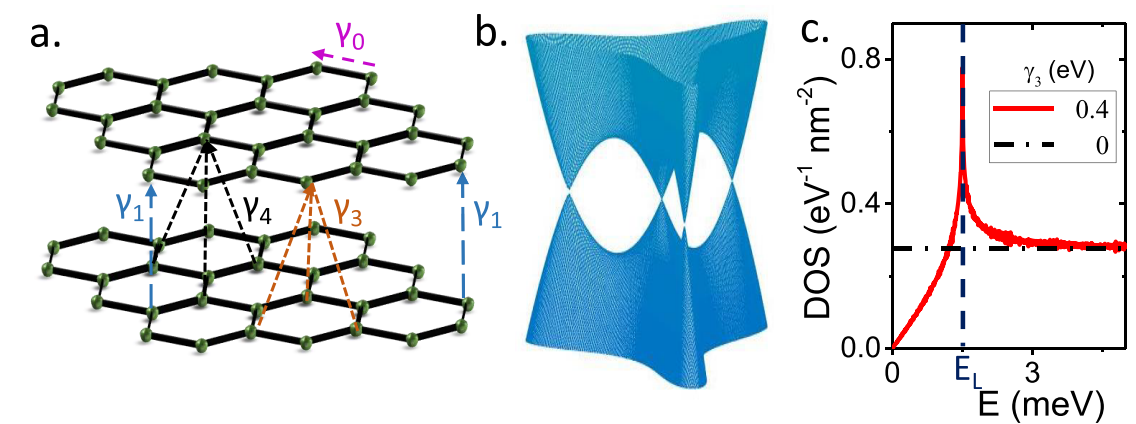

Figure 1. Lifshitz transition in BLG. (a) Schematic of lattice structure of Bernal-stacked BLG. The different electron hopping terms are shown. (b) Band structure of BLG showing the trigonal distortion of the Fermi surface. (c) DOS of BLG with the inclusion of $\gamma_{3}$ showing LT at energy $E_{\mathrm{L}}$. The black dashed line represents the constant DOS in the absence of $\gamma_{3}$ in the Hamiltonian.

a.
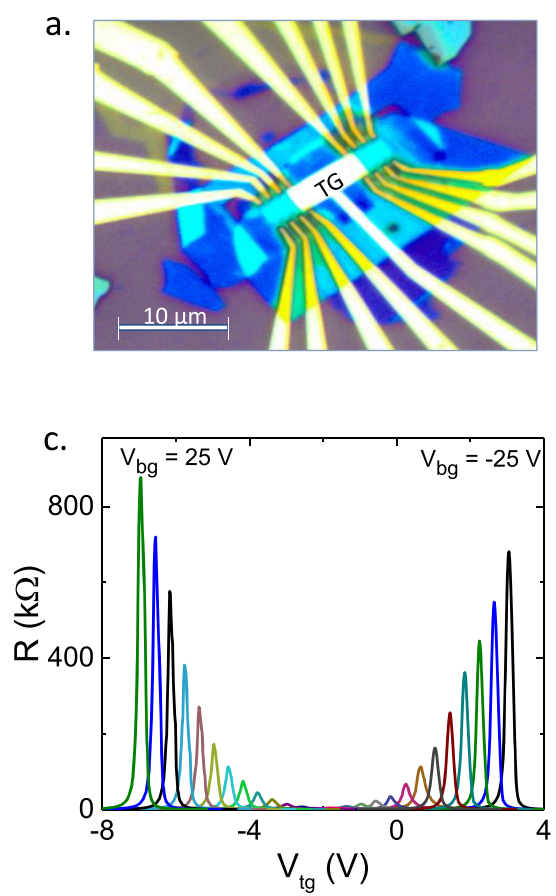
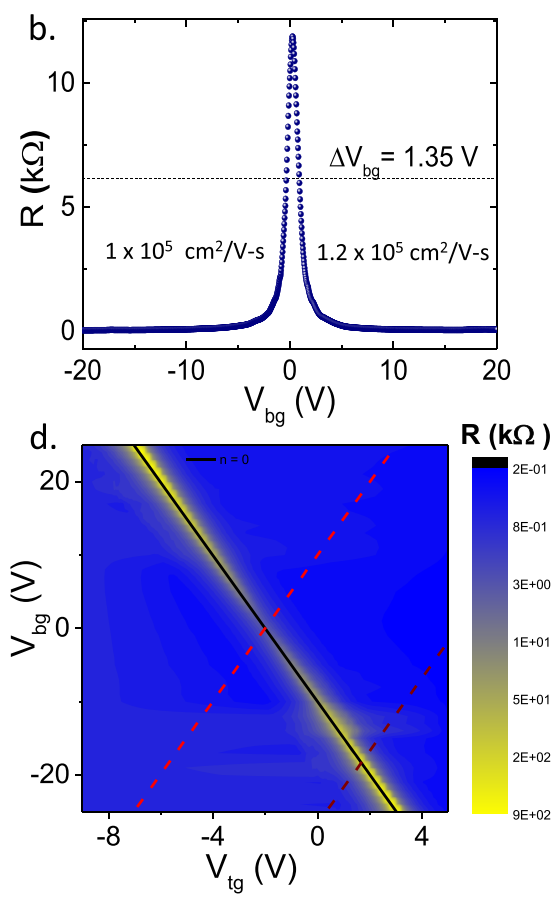

Figure 2. Device structure and characterization. (a) The optical micrograph of the hBN-encapsulated BLG FET device with edge contacts. (b) $R-$ $V_{\mathrm{bg}}$ at $8 \mathrm{~K}$ with mobilities $\mu_{\mathrm{h}}=1 \times 10^{5} \mathrm{~cm}^{2} / \mathrm{V} \cdot \mathrm{s}$ and $\mu_{e}=1.2 \times 10^{5} \mathrm{~cm}^{2} / \mathrm{V} \cdot \mathrm{s}$. The FWHM of $R-V_{\mathrm{bg}}$ is $1.35 \mathrm{~V}$. (c) Transfer characteristics of the device at different values of $V_{\mathrm{bg}}$ from -25 to $25 \mathrm{~V}$ in steps of $2 \mathrm{~V}(T=8 \mathrm{~K})$ (d) Color plot of resistance in the $\left(V_{\mathrm{bg}}, V_{\mathrm{tg}}\right)$ phase space.

where $\Delta V$ is the thermoelectric voltage, $G$ is the energydependent conductance of the sample, $T$ is the sample temperature, and $\Delta T$ is the temperature gradient. Importantly, the factor $\mathrm{d} G / \mathrm{d} n$ is evaluated from the experimentally obtained transfer characteristics and therefore captures the TEP contributions from energy-dependent and energy-independent scattering processes (section S12). From the Mott relation, it is evident that small changes in the conductance lead to large changes in the TEP since it is proportional to the derivative of the DOS within the semiclassical Boltzmann theory. Our experiments reveal new features observed in the TEP that can be attributed to trigonal warping, which is enhanced due to the application of a transverse electric field and the inbuilt sample strain, thereby confirming the TEP as an ideal transport coefficient to resolve low-energy features in the DOS.

\section{RESULTS AND DISCUSSION}

The boron nitride (hBN)-encapsulated BLG device was fabricated in a dual-gated geometry to have independent control over both the band gap and the number density. Figure 2 a shows the optical image of a BLG dual-gated field-effect transistor (FET) device (data from another device with similar characteristics are presented in the Supporting Information Section S2). To study the intrinsic channel characteristics with minimal effects from electrical contacts, the probes were deposited by an edge-contacting method as described in ref 23 . The local top gate (Figure $2 \mathrm{a}$ ) covers the main channel (6.5 $\mu \mathrm{m})$, whereas the channels outside the main channel are gated by the global $\mathrm{SiO}_{2} / \mathrm{Si}^{++}$back gate alone. The graphene channel outside the top-gated region is used as a heater to set up a temperature gradient across the main channel for thermoelectric measurements (see Figure 3a).

Figure $2 \mathrm{~b}$ represents the back-gate transfer characteristics of the device. The extracted field-effect mobility of the channel at $8 \mathrm{~K}$ is $\mu_{\mathrm{FE}} \approx 1.2 \times 10^{5} \mathrm{~cm}^{2} /(\mathrm{V} \cdot \mathrm{s})$. The width of the resistance versus the gate voltage peak at the CNP gives an estimate of the charge inhomogeneity resulting from electron-hole puddle formation at low densities. ${ }^{24}$ In Figure $2 b$, the full-width at half-maximum (FWHM) of $R\left(V_{\mathrm{bg}}\right)$ is $\sim 1.35 \mathrm{~V}$, giving an upper 
bound for the disorder-induced carrier density fluctuation of $\delta n \lesssim 9 \times 10^{10} \mathrm{~cm}^{-2}$. The higher mobility of the sample and the smaller value of $\delta n$ signify the minimal presence of external disorder and impurities. Figure $2 \mathrm{c}$ shows the transfer characteristics of the dual-gated BLG device. The top gate was swept by keeping the back gate fixed at different values between -25 and $25 \mathrm{~V}$. The phase-space color plot of the same data is plotted in Figure $2 \mathrm{~d}$. The solid-black diagonal line corresponds to positions of the CNP at various values of the electric field $D(n=0)$, while the dashed lines correspond to the locus of $D=0$ (red) and $-0.1 \mathrm{~V} / \mathrm{nm}$ (brown).

The TEP was obtained from the ratio of the thermoelectric voltage to the temperature gradient $\left(V_{2 \omega} / \Delta T\right)$ across the channel. The measurement schematic for the TEP is presented in Figure 3a. A temperature gradient is generated across the channel via Joule heating by passing a sinusoidal current $\left(I_{\omega}\right)$ along a channel outside the top-gated region. The resulting second-harmonic thermoelectric voltage $\left(V_{2 \omega}\right)$ was measured across the top-gated channel at different transverse electric fields $(D)$ with varying number densities. Since the temperature gradient set up is proportional to the Joule heating $\left(\propto I^{2}\right)$, $V_{2 \omega}$ also scales with $I_{\omega}{ }^{2}$ for the range of heating currents used (100-600 nA), ensuring that the TEP measurements are performed in the linear response regime $(\Delta T \ll T)$. This is confirmed in Figure $3 \mathrm{~b}$ and its inset.

Since the thermopower measurements are done at low temperatures $(8 \mathrm{~K})$, graphene-resistance thermometry is not a useful method to estimate the value of $\Delta T$ because of the weak dependence of the graphene resistance on temperature. ${ }^{25}$ Hence, we estimated $\Delta T$ by fitting the Mott formula to the measured thermovoltage $V_{2 \omega}$ at $D=0$. Figure $3 c$ shows the Mott fitting for $V_{2 \omega}\left(D=0 \mathrm{~V} / \mathrm{nm}\right.$ and $\left.I_{\mathrm{h}}=500 \mathrm{nA}\right)$ with $\Delta T$ as a fitting parameter. The fit gives $\Delta T \approx 0.25 \mathrm{~K}$. Similar Mott fits are obtained for different values of $I_{\mathrm{h}}$, and the extracted values of $\Delta T$ are plotted versus $I_{\mathrm{h}}{ }^{2}$ in the inset of Figure $3 \mathrm{c}$. The linearity of the estimated values of $\Delta T$ confirms the selfconsistency of this method. This method of determining $\Delta T$ is justified under the assumption that the system obeys the Mott relation, which is indeed true for BLG. ${ }^{20,26}$ The same calibration of $\Delta T$ was subsequently used for the thermopower estimation at a non-zero value $D$ upon scaling with the corresponding heater resistance (see Figure S6). The calculated $S$ values for different heating currents (with the same calibration of $\Delta T$ ) collapse on top of one another, providing additional support to the method (insets in Figure 3d).

Figure $3 \mathrm{~d}$ shows the evolution of the thermoelectric voltage, with an increasing gap for different values of the heating current $(100-600 \mathrm{nA})$. Previous reports on gapped $\mathrm{BLG}^{20,27}$ show a considerable enhancement in the thermopower as a function of $D$, which is consistent with our observation (Figure S7). This enhancement is due to the change in the band curvature at band edges with increasing values of $D .^{20}$ Moreover, increasing the value of $D$ introduces sharp fluctuations in the thermopower when the chemical potential is within the gap. These fluctuations occur very close to $n=0$, and the gate voltage window over which the fluctuations occur, increases with the opening of the band gap. Upon closer inspection, these fluctuations seem to be reproducible, with multiple sweeps overlapping on top of one another (Figure $3 \mathrm{e})$. Universal conductance fluctuations can be ruled out because the channel is strongly localized in this gate voltage window $\left(G \ll e^{2} / h\right)$. Localized state transport in gapped BLG has been investigated in several recent reports, ${ }^{25,28-31}$ where the possibility of variable range hopping has been discussed. Due to the marginal topology of bilayer graphene, such hopping conduction has been suggested to be quasi-onedimensional in nature. ${ }^{32-34}$ The multiple-sweep reproducibility of fluctuations in the $V_{\mathrm{tg}}$ dependence of both $V_{2 \omega}$ and conductance (Figure $3 \mathrm{e}$ ) is strongly indicative of a hopping transport mediated by midgap states due to frozen disorder in our gapped BLG devices (see the Supporting Information Section S4 for more details). ${ }^{32,35}$

The new and most striking observation in our measurements is the presence of thermopower "plateaus" in the vicinity of the CNP (marked by arrows in Figure 3d). These highly reproducible features, present on either side of the $\mathrm{CNP}$, are reflected only in the TEP, with no corresponding features in the resistance measurements (Figure S8). The plateaus, although not visible for $D=0 \mathrm{~V} / \mathrm{nm}$, were observed for all measured values of $D>0$, and the density at which the plateaus occur is weakly dependent on $D$. They occur at $n_{\mathrm{L}} \approx$ $2.5 \times 10^{11} \mathrm{~cm}^{-2}$ and $n_{\mathrm{L}} \approx-3.3 \times 10^{11} \mathrm{~cm}^{-2}$ on the electron and hole sides respectively, for all values of $D$. These plateaus are reminiscent of "entropy spikes", expected to occur at LTs in $2 \mathrm{D}$ systems such as germanene, silicene, and SLG. ${ }^{36-38}$ Recent theoretical studies have also predicted the occurrence of similar secondary maxima in the thermopower as a function of density in ballistic BLG system because of the LT. ${ }^{39,40}$

To interpret the presence of the plateaus in TEP measurements at a characteristic number density, tight-binding calculations of BLG with the inclusion of trigonal warping were performed. The four-band effective Hamiltonian for BLG for low energy excitations is given by ${ }^{12}$

$$
\hat{H}=\left[\begin{array}{cccc}
\frac{\xi}{2} U & v_{3} \hat{\pi}+w & 0 & v_{\mathrm{f}} \hat{\pi}^{\dagger} \\
v_{3} \hat{\pi}^{\dagger}+w^{*} & -\frac{\xi}{2} U & v_{\mathrm{f}} \hat{\pi} & 0 \\
0 & v_{\mathrm{f}} \hat{\pi}^{\dagger} & -\frac{\xi}{2} U & \xi \gamma_{1} \\
v_{\mathrm{f}} \hat{\pi} & 0 & \xi \gamma_{1} & \frac{\xi}{2} U
\end{array}\right]
$$

where, $\xi=1(\xi=-1)$ refers to the $K\left(K^{\prime}\right)$ valley, $\gamma_{1}(0.381 \mathrm{eV})$ is the interlayer coupling, $\gamma_{3}(0.4 \mathrm{eV})$ is the skew interlayer coupling (see the Supporting Information Section S3), $U$ is the interlayer asymmetry term, and $\hat{\pi}=p_{x}+i p_{y}$. Here, $v_{3}=\frac{\sqrt{3} a}{2 \hbar} e \gamma_{3}$, where $a$ is the lattice constant of BLG and $w$ is a complex gauge parameter that represents the magnitude of the homogeneous strain on BLG. ${ }^{17}$

The addition of a skew interlayer hopping term also modifies the low-energy band structure of gapped BLG. Unlike the fragmentation of the Fermi surface into four pockets as in the case of BLG at $D=0 \mathrm{~V} / \mathrm{nm}$, the conduction and valence bands are trigonally distorted near the band edge with three disconnected Fermi pockets, as shown in Figure $4 \mathrm{a}$ for $D=$ $0.4 \mathrm{~V} / \mathrm{nm}$. Therefore, the interplay of interlayer asymmetry and trigonal warping produces a LT close to the band edge, as shown in the DOS plot (Figure $4 \mathrm{c}$ (brown)). The value of $n_{\mathrm{L}}$ is calculated by integrating the DOS over the energy range from 0 to $E_{\mathrm{L}}$. Figure $4 \mathrm{~d}$ shows the calculated values of $n_{\mathrm{L}}$ as compared to the experimentally observed values. Clearly, the calculated values of $n_{\mathrm{L}}$ (red trace) do not match the 


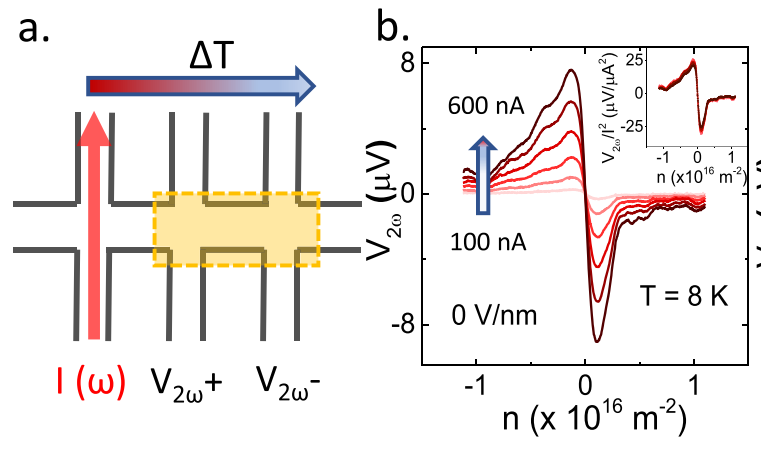

C.

d.
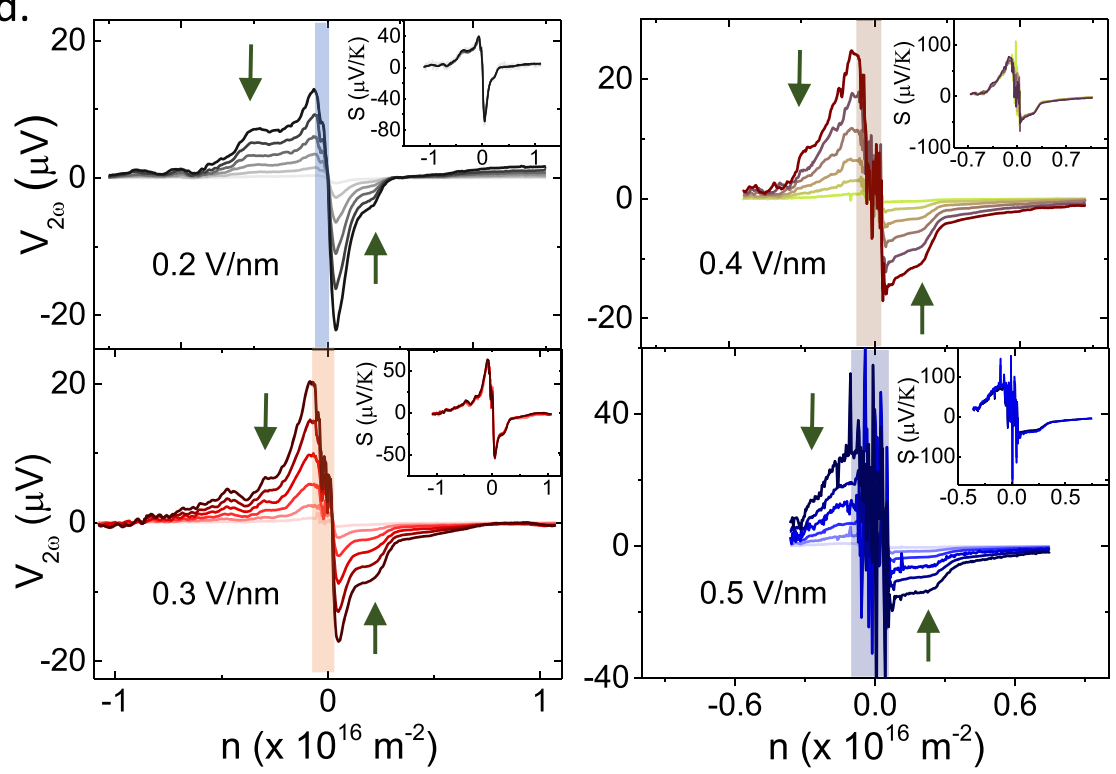

e.

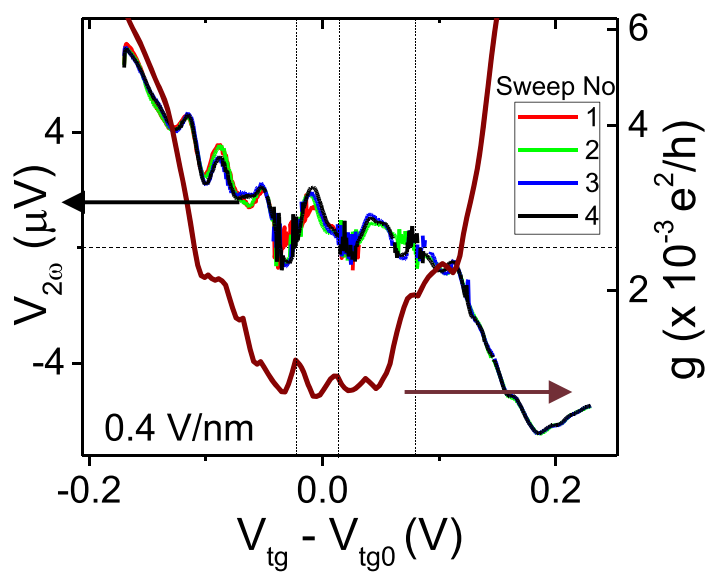

Figure 3. Thermoelectric measurements. (a) Contact configuration for thermopower measurements. (b) $V_{2 \omega}$ as a function of the number density $n$ for different values of heating currents at $8 \mathrm{~K}$ and $D=0 \mathrm{~V} / \mathrm{nm}$. The inset shows the normalized curve of $V_{2 \omega} / I^{2}$ vs $n$. (c) Mott fitting of $V_{2 \omega}\left(I_{\mathrm{h}}=\right.$ $500 \mathrm{nA}$ ) with $\Delta T$ as a fitting parameter. The extracted values of $\Delta T$ at various heating currents are presented in the inset. (d) Density dependence of the thermovoltage at different values of $D(T=8 \mathrm{~K})$ for various heating currents. The shaded portion indicates the region of fluctuating thermopower. Insets show the thermopower calculated for a non-zero $D$, with the same calibration of $\Delta T$ estimated for $D=0$ for different heating currents. (e) Magnified version of $V_{2 \omega}$ near the CNP for $D=0.4 \mathrm{~V} / \mathrm{nm}$ showing the repeatability of the fluctuations (shaded box in panel d). The corresponding fluctuations in the conductance are also shown.

experimental values and show a monotonous increase with $D$ in contrast to a relatively invariant value obtained in the experiment. Therefore, a simple addition of the trigonal warping term fails to explain the weak dependence of $n_{\mathrm{L}}$ with D.

Multiple theoretical reports predict the enhancement of $E_{\mathrm{L}}$ with strain. ${ }^{17,41}$ Strain can be induced either intentionally or unintentionally due to the presence of a mechanical deformation of the sample, either by a uniaxial strain or an interlayer shear shift caused during sample preparation. ${ }^{17,41,42}$ Graphene-hBN van der Waals heterostructures are known to host strain of $\sim 0.6-1 \%{ }^{43,44}$ because of thermal cycling, local corrugations like bubbles, or lattice commensuration. ${ }^{45}$ In addition, epitaxially grown graphene-hBN heterostructures can 
host strain up to $1.8 \%$ because of lattice matching with $\mathrm{hBN}{ }^{46}$ To estimate the built-in strain in our devices, Raman measurements were performed on an identically fabricated BLG sample, which showed a significant shift in the G and 2D peaks ( 9 and $20 \mathrm{~cm}^{-1}$, respectively) that indicated the presence of strain (Figure S12). The large shift in the 2D peak rules out the possibility of shift due to charge doping. ${ }^{43}$ The shift corresponds to a uniaxial strain with a magnitude of $\sim 0.4-$ 0.7\% (see the Supporting Information Section S11).

Theoretically, this strain can be introduced into the band structure by incorporating a complex parameter $w$ in the Hamiltonian (eq 2). It has been estimated that $1 \%$ strain in monolayer graphene results in $|w| \sim 6 \mathrm{meV} .^{17}$ Figure $4 \mathrm{~b}$ represents the band structure of BLG with the inclusion of strain $(w=6 i \mathrm{meV})$. Strain-induced deformation of the band structure gives rise to distinctive features in the DOS. The addition of strain breaks the symmetry between the side cones, resulting in multiple saddle points and parabolic minima that give rise to multiple Lifshitz transitions as shown Figure $4 \mathrm{c}$ (shaded region). It has to be noted that the strain-induced spectral changes are very sensitive to the parameter $w$ (Figure S9). The calculated values of $n_{\mathrm{L}}$ obtained upon the inclusion of strain $(w=6 i \mathrm{meV})$ are nearly invariant for $D>0$ (blue trace in Figure 4c) and match well with the experimental values in the electron side.

Panels e and $\mathrm{f}$ in Figure 4 show the fitting of the Mott formula (eq 1) of the data calculated with the inclusion of both $\gamma_{3}$ and $w$ in the DOS for $D=0.3$ and $0.4 \mathrm{~V} / \mathrm{nm}$, respectively. It shows an excellent fit and reproduces the plateau in the electron-doped region (Figure $4 \mathrm{~g}$ ). The Mott fits calculated without these modifications in the DOS are represented with dashed lines, and they fail to reproduce the plateaus. This "apparent" violation of the Mott formula is resolved with the inclusion of $\gamma_{3}$ and strain, indicating that the plateaus originate due to strain-enhanced trigonal warping. Although the addition of strain replicates the plateaus in the hole-doped region as well, the calculated value of $n_{\mathrm{L}}$ is electron-hole symmetric and hence does not match the experimental value for $n<0$, possibly because of inhomogeneneity in the strain that is not considered in our calculations. Similar fittings of the Mott relation have been performed for other values of $D$ (Figure S10). Although the addition of strain in the form of a gauge field $(w)$ quantitatively describes the plateaus, correlation effects were neglected in our calculation and cannot be discounted. A Coulomb interaction is predicted to further enhance the strain-induced effects, leading to a larger value of $w$ even for negligible values of strain, which could explain the overestimation of the value of strain used in the model. ${ }^{17}$ The interaction-driven reconstruction of the low-energy band structure could also explain the larger Lifshitz energy $E_{\mathrm{L}} \cdot{ }^{13}$

Finally, Figure 5a shows the temperature dependence of the TEP at $D=0.4 \mathrm{~V} / \mathrm{nm}$. While the plateaus are smoothened over and are not observed beyond $17 \mathrm{~K}$, there is no evidence of these features at $77 \mathrm{~K}$ because of temperature-induced broadening of the DOS. The absence of plateaus in the case of $D=0 \mathrm{~V} / \mathrm{nm}$ can also be explained due to the lower value of $n_{\mathrm{L}}$ (Figure $4 \mathrm{c}$ ), resulting in the smearing of the plateau even at $8 \mathrm{~K}$. There is no evidence of strain relaxation effects with increasing temperature since the plateaus occur at approximately the same number density for all values of $T$ (shaded region in Figure 5a). Figure $5 \mathrm{~b}$ shows the Mott fitting of $V_{2 \omega}$ at $\mathrm{D}=0.4 \mathrm{~V} / \mathrm{nm}$ at $77 \mathrm{~K}$, which shows a reasonably good fit even without the addition of $\gamma_{3}$ or strain and indicates the inability to resolve the low-energy features at higher temperatures.

In conclusion, we demonstrate the use of thermoelectric measurements as an alternative probe to investigate the fine details of the band structure in BLG. We observe reproducible
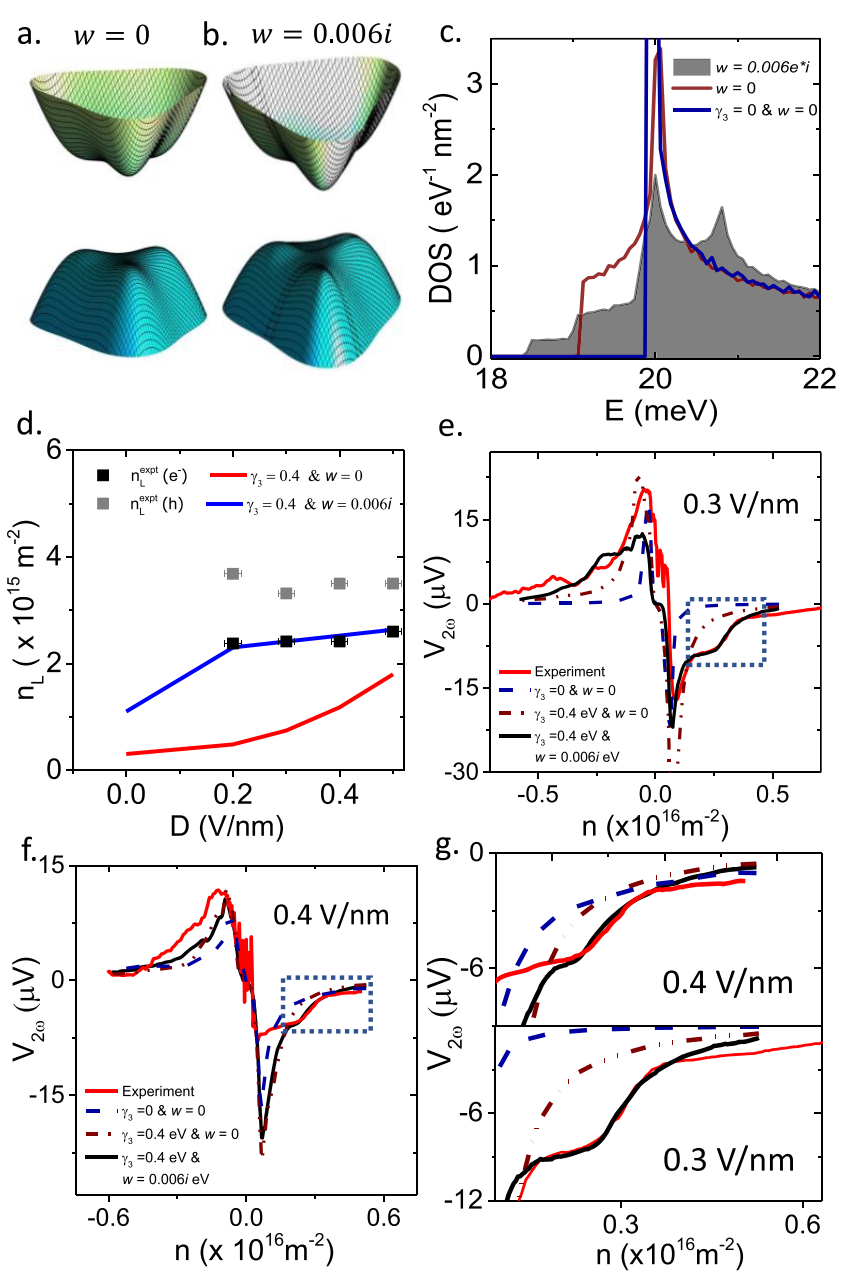

Figure 4. Mott fitting of the TEP. Band structure of BLG with $\gamma_{3}=$ $0.4 \mathrm{eV}$ and $D=0.4 \mathrm{~V} / \mathrm{nm}$ for $w=$ (a) 0 and (b) $6 i \mathrm{meV}$. (c) DOS of BLG $(D=0.4 \mathrm{~V} / \mathrm{nm})$. The shaded region shows the DOS with the inclusion of strain. (d) Calculated $n_{\mathrm{L}}$ vs $D$ compared with the experimental data. The error bar corresponds to the variation in $D$ upon sweeping $V_{\mathrm{tg}}$ while keeping $V_{\mathrm{bg}}$ constant. Mott fitting of TEP with various approximations of DOS for $D=$ (e) 0.3 and (f) $0.4 \mathrm{~V} /$ $\mathrm{nm}$. $(\mathrm{g})$ Magnified version of panels e and $\mathrm{f}$ to show the fitting of the Mott formula in the electron-doped region.
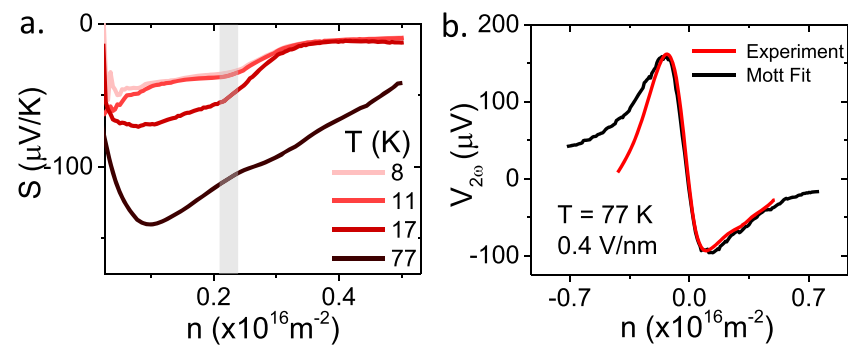

Figure 5. Temperature dependence. (a) Plot of the TEP vs $n$ at various temperatures for $D=0.4 \mathrm{~V} / \mathrm{nm}$. (b) Mott fitting of the TEP for $D=0.4 \mathrm{~V} / \mathrm{nm}$ at $77 \mathrm{~K}\left(\gamma_{3}=0\right.$ and $\left.w=0\right)$. 
thermopower fluctuations within the band gap of BLG. In addition, the TEP in high-mobility devices enables us to detect possible evidence of a Lifshitz transition enhanced by the presence of strain as well as band gap, which is not easily captured in conductance measurements.

\section{ASSOCIATED CONTENT}

\section{SI Supporting Information}

The Supporting Information is available free of charge at https://pubs.acs.org/doi/10.1021/acs.nanolett.0c03586.

Methods, results from device 2, value of $\gamma_{3}$, transport characteristics, $\Delta T$ calibration, thermopower enhancement as a function of $D$, comparison of the TEP with conductance, sensitivity of the DOS to the complex strain parameter $(w)$, Mott fits, electron-hole symmetry-breaking interlayer hopping, $\gamma_{4}$, estimation of built-in strain with Raman spectroscopy, and energy-dependent scattering to the thermopower-near Lifshitz transition (PDF)

\section{AUTHOR INFORMATION}

\section{Corresponding Author}

Aditya Jayaraman - Department of Physics, Indian Institute of Science, Bangalore 560012, India; 이이. orcido000-00026963-7195; Email: jaditya@iisc.ac.in

\section{Authors}

Kimberly Hsieh - Department of Physics, Indian Institute of Science, Bangalore 560012, India; (1) orcid.org/0000-00017229-9636

Bhaskar Ghawri - Department of Physics, Indian Institute of Science, Bangalore 560012, India

Phanibhusan S. Mahapatra - Department of Physics, Indian Institute of Science, Bangalore 560012, India; (1) orcid.org/ 0000-0002-1070-2116

Kenji Watanabe - Research Center for Functional Materials, National Institute for Materials Science, Tsukuba 305-0044, Japan; (i) orcid.org/0000-0003-3701-8119

Takashi Taniguchi - International Center for Materials Nanoarchitectonics, National Institute for Materials Science, Tsukuba 305-0044, Japan; 이이.org/0000-0002-14673105

Arindam Ghosh - Department of Physics, Indian Institute of Science, Bangalore 560012, India; Centre for Nano Science and Engineering, Indian Institute of Science, Bangalore 560012, India

Complete contact information is available at:

https://pubs.acs.org/10.1021/acs.nanolett.0c03586

\section{Notes}

The authors declare no competing financial interest.

\section{ACKNOWLEDGMENTS}

This work was supported by the Department of Science and Technology, Government of India. A.J. and A.G. thank the National Nanofabrication Center, CeNSE, IISc (NNfC) for providing clean room facilities as well as the Micro and Nano Characterization Facility, CeNSE, IISc (MNCF) for Raman characterization. We would like to thank Dr. Manjari Garg and Prof. Subroto Mukerjee for valuable discussions. K.W. and T.T. acknowledge support from the Elemental Strategy Initiative conducted by the MEXT, Japan, Grant JPMXP0112101001,
JSPS KAKENHI Grant JP20H00354, and the CREST(no. JPMJCR15F3), JST.

\section{REFERENCES}

(1) Lifshitz, I.; et al. Anomalies of electron characteristics of a metal in the high pressure region. Sov. Phys. JETP 1960, 11 (5), 1130-1135.

(2) Norman, M. R.; Lin, J.; Millis, A. J. Lifshitz transition in underdoped cuprates. Phys. Rev. B: Condens. Matter Mater. Phys. 2010, 81,180513

(3) Shi, X.; Han, Z.; Peng, X.; Richard, P.; Qian, T.; Wu, X.; Qiu, M.; Wang, S.; Hu, J.; Sun, Y.; et al. Enhanced superconductivity accompanying a Lifshitz transition in electron-doped FeSe monolayer. Nat. Commun. 2017, 8, 14988.

(4) Sandeman, K. G.; Lonzarich, G. G.; Schofield, A. J. Ferromagnetic Superconductivity Driven by Changing Fermi Surface Topology. Phys. Rev. Lett. 2003, 90, 167005.

(5) Yamaji, Y.; Misawa, T.; Imada, M. Quantum metamagnetic transitions induced by changes in Fermi-surface topology: Applications to a weak itinerant-electron ferromagnet $\mathrm{ZrZn2}$. J. Phys. Soc. Jpn. 2007, 76, 063702.

(6) Yelland, E.; Barraclough, J.; Wang, W.; Kamenev, K.; Huxley, A. High-field superconductivity at an electronic topological transition in URhGe. Nat. Phys. 2011, 7, 890.

(7) Yang, H.; Yang, L.; Liu, Z.; Sun, Y.; Chen, C.; Peng, H.; Schmidt, M.; Prabhakaran, D.; Bernevig, B. A.; Felser, C.; et al. Topological Lifshitz transitions and Fermi arc manipulation in Weyl semimetal NbAs. Nat. Commun. 2019, 10, 3478.

(8) $\mathrm{Xu}, \mathrm{N}$.; et al. Evidence of a Coulomb-Interaction-Induced Lifshitz Transition and Robust Hybrid Weyl Semimetal in $T_{d}-$ $\mathrm{MoTe}_{2}$. Phys. Rev. Lett. 2018, 121, 136401.

(9) Barber, M. E.; Gibbs, A. S.; Maeno, Y.; Mackenzie, A. P.; Hicks, C. W. Resistivity in the Vicinity of a van Hove Singularity: $\mathrm{Sr}_{2} \mathrm{RuO}_{4}$ under Uniaxial Pressure. Phys. Rev. Lett. 2018, 120, 076602.

(10) Nishimura, T.; Sakai, H.; Mori, H.; Akiba, K.; Usui, H.; Ochi, M.; Kuroki, K.; Miyake, A.; Tokunaga, M.; Uwatoko, Y.; et al. Large Enhancement of Thermoelectric Efficiency Due to a Pressure-Induced Lifshitz Transition in SnSe. Phys. Rev. Lett. 2019, 122, 226601.

(11) McCann, E.; Abergel, D. S.; Fal'ko, V. I. The low energy electronic band structure of bilayer graphene. Eur. Phys. J.: Spec. Top. 2007, 148, 91-103.

(12) McCann, E.; Koshino, M. The electronic properties of bilayer graphene. Rep. Prog. Phys. 2013, 76, 056503.

(13) Mayorov, A.; Elias, D.; Mucha-Kruczynski, M.; Gorbachev, R.; Tudorovskiy, T.; Zhukov, A.; Morozov, S.; Katsnelson, M.; Fal'ko, V.; Geim, A.; et al. Interaction-driven spectrum reconstruction in bilayer graphene. Science 2011, 333, 860-863.

(14) Varlet, A.; Bischoff, D.; Simonet, P.; Watanabe, K.; Taniguchi, T.; Ihn, T.; Ensslin, K.; Mucha-Kruczyński, M.; Fal'ko, V. I. Anomalous sequence of quantum hall liquids revealing a tunable Lifshitz transition in bilayer graphene. Phys. Rev. Lett. 2014, 113, 116602.

(15) Oka, T.; Tajima, S.; Ebisuoka, R.; Hirahara, T.; Watanabe, K.; Taniguchi, T.; Yagi, R. Ballistic transport experiment detects Fermi surface anisotropy of graphene. Phys. Rev. B: Condens. Matter Mater. Phys. 2019, 99, 035440.

(16) Joucken, F.; Ge, Z.; Quezada-López, E. A.; Davenport, J. L.; Watanabe, K.; Taniguchi, T.; Velasco, J., Jr Determination of the trigonal warping orientation in Bernal-stacked bilayer graphene via scanning tunneling microscopy. Phys. Rev. B: Condens. Matter Mater. Phys. 2020, 101, 161103.

(17) Mucha-Kruczyński, M.; Aleiner, I. L.; Fal'ko, V. I. Strained bilayer graphene: Band structure topology and Landau level spectrum. Phys. Rev. B: Condens. Matter Mater. Phys. 2011, 84, 041404.

(18) Dollfus, P.; Nguyen, V. H.; Saint-Martin, J. Thermoelectric effects in graphene nanostructures. J. Phys.: Condens. Matter 2015, 27, 133204.

(19) Zuev, Y. M.; Chang, W.; Kim, P. Thermoelectric and magnetothermoelectric transport measurements of graphene. Phys. Rev. Lett. 2009, 102, 096807. 
(20) Wang, C.-R.; Lu, W.-S.; Hao, L.; Lee, W.-L.; Lee, T.-K.; Lin, F.; Cheng, I.-C.; Chen, J.-Z. Enhanced thermoelectric power in dualgated bilayer graphene. Phys. Rev. Lett. 2011, 107, 186602.

(21) Mahapatra, P. S.; Sarkar, K.; Krishnamurthy, H.; Mukerjee, S.; Ghosh, A. Seebeck coefficient of a single van der Waals junction in twisted bilayer graphene. Nano Lett. 2017, 17, 6822-6827.

(22) Mahapatra, P. S.; Ghawri, B.; Garg, M.; Mandal, S.; Watanabe, K.; Taniguchi, T.; Jain, M.; Mukerjee, S.; Ghosh, A. MisorientationControlled Cross-Plane Thermoelectricity in Twisted Bilayer Graphene. Phys. Rev. Lett. 2020, 125, 226802.

(23) Wang, L.; Meric, I.; Huang, P.; Gao, Q.; Gao, Y.; Tran, H.; Taniguchi, T.; Watanabe, K.; Campos, L.; Muller, D.; et al. Onedimensional electrical contact to a two-dimensional material. Science 2013, 342, 614-617.

(24) Dean, C. R.; Young, A. F.; Meric, I.; Lee, C.; Wang, L.; Sorgenfrei, S.; Watanabe, K.; Taniguchi, T.; Kim, P.; Shepard, K. L.; et al. Boron nitride substrates for high-quality graphene electronics. Nat. Nanotechnol. 2010, 5, 722.

(25) Taychatanapat, T.; Jarillo-Herrero, P. Electronic transport in dual-gated bilayer graphene at large displacement fields. Phys. Rev. Lett. 2010, 105, 166601.

(26) Nam, S.-G.; Ki, D.-K.; Lee, H.-J. Thermoelectric transport of massive Dirac fermions in bilayer graphene. Phys. Rev. B: Condens. Matter Mater. Phys. 2010, 82, 245416.

(27) Chien, Y.-Y.; Yuan, H.; Wang, C.-R.; Lee, W.-L. Thermoelectric power in bilayer graphene device with ionic liquid gating. Sci. Rep. 2016, 6, 20402.

(28) Zou, K.; Zhu, J. Transport in gapped bilayer graphene: The role of potential fluctuations. Phys. Rev. B: Condens. Matter Mater. Phys. 2010, 82, 081407.

(29) Miyazaki, H.; Tsukagoshi, K.; Kanda, A.; Otani, M.; Okada, S. Influence of disorder on conductance in bilayer graphene under perpendicular electric field. Nano Lett. 2010, 10, 3888.

(30) Yan, J.; Fuhrer, M. S. Charge transport in dual gated bilayer graphene with corbino geometry. Nano Lett. 2010, 10, 4521.

(31) Oostinga, J. B.; Heersche, H. B.; Liu, X.; Morpurgo, A. F.; Vandersypen, L. M. K. Gate-induced insulating state in bilayer graphene devices. Nat. Mater. 2008, 7, 151.

(32) Aamir, M. A.; Karnatak, P.; Jayaraman, A.; Sai, T. P.; Ramakrishnan, T.; Sensarma, R.; Ghosh, A. Marginally self-averaging one-dimensional localization in bilayer graphene. Phys. Rev. Lett. 2018, 121, 136806.

(33) Li, J.; Martin, I.; Büttiker, M.; Morpurgo, A. F. Marginal topological properties of graphene: a comparison with topological insulators. Phys. Scr. 2012, 2012 (T146), 014021.

(34) Li, J.; Martin, I.; Büttiker, M.; Morpurgo, A. F. Topological origin of subgap conductance in insulating bilayer graphene. Nat. Phys. 2011, 7, 38.

(35) Hughes, R.; Savchenko, A.; Frost, J.; Linfield, E.; Nicholls, J.; Pepper, M.; Kogan, E.; Kaveh, M. Distribution-function analysis of mesoscopic hopping conductance fluctuations. Phys. Rev. B: Condens. Matter Mater. Phys. 1996, 54, 2091.

(36) Galperin, Y.; Grassano, D.; Gusynin, V.; Kavokin, A.; Pulci, O.; Sharapov, S.; Shubnyi, V.; Varlamov, A. Entropy Signatures of Topological Phase Transitions. J. Exp. Theor. Phys. 2018, 127, 958983.

(37) Grassano, D.; Pulci, O.; Shubnyi, V.; Sharapov, S.; Gusynin, V.; Kavokin, A.; Varlamov, A. Detection of topological phase transitions through entropy measurements: The case of germanene. Phys. Rev. B: Condens. Matter Mater. Phys. 2018, 97, 205442.

(38) Sharapov, S.; Varlamov, A. Anomalous growth of thermoelectric power in gapped graphene. Phys. Rev. B: Condens. Matter Mater. Phys. 2012, 86, 035430.

(39) Suszalski, D.; Rut, G.; Rycerz, A. Lifshitz transition and thermoelectric properties of bilayer graphene. Phys. Rev. B: Condens. Matter Mater. Phys. 2018, 97, 125403.

(40) Suszalski, D.; Rut, G.; Rycerz, A. Thermoelectric properties of gapped bilayer graphene. J. Phys.: Condens. Matter 2019, 31, 415501.
(41) Varlet, A.; Mucha-Kruczyński, M.; Bischoff, D.; Simonet, P.; Taniguchi, T.; Watanabe, K.; Fal'ko, V.; Ihn, T.; Ensslin, K. Tunable Fermi surface topology and Lifshitz transition in bilayer graphene. Synth. Met. 2015, 210, 19-31.

(42) Davydov, V. Some peculiarities of thermopower at the Lifshitz topological transitions due to stacking change in bilayer and multilayer graphene. Proc. R. Soc. London, Ser. A 2019, 475, 20190028.

(43) Pan, W.; Xiao, J.; Zhu, J.; Yu, C.; Zhang, G.; Ni, Z.; Watanabe, K.; Taniguchi, T.; Shi, Y.; Wang, X. Biaxial compressive strain engineering in graphene/boron nitride heterostructures. Sci. Rep. 2012, 2, 893.

(44) Vincent, T.; Panchal, V.; Booth, T.; Power, S. R.; Jauho, A.-P.; Antonov, V.; Kazakova, O. Probing the nanoscale origin of strain and doping in graphene-hBN heterostructures. 2D Mater. 2019, 6, 015022 .

(45) Woods, C.; Britnell, L.; Eckmann, A.; Ma, R.; Lu, J.; Guo, H.; Lin, X.; Yu, G.; Cao, Y.; Gorbachev, R.; et al. Commensurateincommensurate transition in graphene on hexagonal boron nitride. Nat. Phys. 2014, 10, 451-456.

(46) Davies, A.; Albar, J. D.; Summerfield, A.; Thomas, J. C.; Cheng, T. S.; Korolkov, V. V.; Stapleton, E.; Wrigley, J.; Goodey, N. L.; Mellor, C. J.; et al. Lattice-matched epitaxial graphene grown on boron nitride. Nano Lett. 2018, 18, 498-504.

\section{NOTE ADDED AFTER ASAP PUBLICATION}

Figures 2 and 3 were the wrong graphics in the version published January 27, 2021; the corrected version was reposted January 29, 2021. 